\title{
La inteligencia organizacional en la industria biofarmacéutica
}

\section{Eduardo Orozco Silva}

\section{Resumen}

Se presenta un análisis de i estado dej arte de la inteligencia organizacional (empresarial, corporativa, competitiva, económica) desde el punto de vista de su reflejo en la literatura científica de ciencias de la información, de la prensa económica y de la información científica y corporativa sobre tecnologías de información y se discuten varios enfoques aparentemente divergentes. Se valoran los resultados anteriores en relación con su presencia en la industria biofarmacéutica a nivel mundial. Se presenta una descripción de los métodos de trabajo y los resultados de Consultoría Biomundi como centro de inteligencia organizacional para la industria biofarmacéutica.

Palavras-chave

Inteligência organizacional; Indústria biofarmacêutica; Literatura científica Tecnologias da informação.
INTRODUCCIÓN

Nuestro modo tradicional de pensar aunque usemos técnicas matemáticas sofisticadas y una impenetrable jerga sociológica-siempre ha percibido los negocios como comprar barato $y$ vender caro. El nuevo enfoque define un negocio como la organización que agrega valor y crea ríqueza". Peter Drucker. La información que necesitan los ejecutivos de hoy, 1995.

Cualquier enfoque actual de la inteligencia organizacional requiere de un esfuerzo primario de definición, en beneficio de los que se asoman al tema por primera vez y encuentran diferentes denominaciones para designar el mismo fenómeno, a la vez que descubren que procesos complementarios son designados con el mismo nombre, lo que implica un grado alto de confusión en la literatura técnica actual sobre el tema. El autor trató el asunto anteriormente (Orozco, E. 1998), por lo que ahora se limitará a retomar lo planteado anteriormente:

"Varios autores han tratado los temas de inteligencia competitiva" e "inteligencia sobre los competidores", ... y monitoreo del medio" ... En la concepción que se tiene de los mísmos, queda claro que forman parte de la llamada "inteligencia corporativa", expresión española dada a la inglesa "business intelligence", que es la más comúnmente utilizada y la más abarcadora. Algunos autores la identifican como "inteligencia empresarial" y otros usan una expresión aún más general: u inteligencia organizacional". Con un enfoque más amplio por su alcance, Cierc, 1997, presenta el concepto de u inteligencia económica". ... entenderemos por "inteligencia corporativa", la capacidad (y la función) de reunir, analizar $y$ diseminar datos, que permite obtener, de manera sistemática y organizada, información relevante sobre el ambiente externo y sobre las condiciones internas de la organización, para la toma de decisiones $y$ la orientación estratégica.... Por tanto, la inteligencia corporativa, ... es una forma específica de expresión de la gestión de información, y comprende a la inteligencia competitiva (que pone el énfasis en el ambiente competitivo, y en conocer los aspectos de la competencia que pueden hacer impacto en las fortalezas y debilidades de la empresa), la inteligencia sobre los competidores (que se orienta fundamentalmente a conocer los competidores, mediante perfiles de los mismos y su actualización) y al monitoreo del medio (el proceso de vigilancia general de todos los segmentos del medio y sus interacciones, para identificar señales de cambio). La inteligencia corporativa es a la vez un proceso (analítico), que transforma datos en conocimiento estratégico relevante y preciso ... y un producto (la información ya procesada) ... En conjunto, la inteligencia corporativa es a la vez un proceso, una función organizacional y un producto, al cual también se le Ilama inteligencia corporativa ... Vista en función de la gestión organizacional, la inteligencia corporativa es una herramienta gerenciai, que retrata el presente, lo analiza y facilita el manejo de juturo, medíante la utilización de fuentes de información y de herramientas propias de las ciencias de la información. Obviamente, ni como proceso, ni como función organizacional, la inteligencia corporativa se debe confundir con el espionaje industrial ni con ninguna forma de inteligencia no 
ética. Aunque el producto pudiese ser similar, los métodos de trabajo de la inteligencia corporativa se basan en la obtención de información por medios legales, ya sea de fuentes públicas o privadas".

En el periodo transcurrido desde que se escribió la cita previa, han aparecido nuevos trabajos, con más diversidad de enfoques y definiciones. Para algunos autores (Dkaki y Dousset, 1998), "inteligencia competitiva" es sinónimo de u monitoreo dej ambiente científico y tecnológico".

A lo anterior, el autor desea sólo agregar dos elementos:

- Primero, que en su visión de hoy, es más correcto hablar de "inteligencia organizacional", por la amplitud de esta expresión, la que quizás logre convertirse en una denominación única aceptable por todos.

- Segundo, que de cualquier manera será la mayor profusión de j uso de una u otra expresión la que haga finalmente definitiva a alguna de ellas. Entonces habrá que encontrar una definición que todos acepten.

\section{LA INTELIGENCIA ORGANIZACIONAL EN LOS RECURSOS MODERNOS DE INFORMACIÓN}

Un análisis somero de la presencia de literatura científica relacionada con los temas de inteligencia organizacional revela lo siguiente:

\section{En la literatura sobre ciencias de la información.}

La literatura científica de ciencias de la información ha recogido muy pocas contribuciones sobre el tema en 32 años, segun refleja un análisis al respecto efectuado a partir de las bases de datos - Information Science Abstracts (ISA)', en el periodo 196698 y Librarv and Information Science Abstracts (LISA)2, en el periodo 19601998.
En primer lugar, ninguna de las bases de datos ha formalizado en sus herramientas linguísticas los términos relativos al trabajo de inteligencia vistos más arriba. ISA contempla "business intelligence" y "strategic intelligence" como descriptores, pero los asigna a un número menor de citas bibliográficas que las que realmente tratan sobre el tema. LISA no contiene en su tesauro ninguno de los términos mencionados. Ello obliga a efectuar las búsquedas por palabras en el texto.

Aun teniendo en cuenta que ninguna de las dos bases de datos es exhaustiva, es evidente que las contribuciones al tema son mínimas, sobre todo si se tiene en cuenta que ese aporte constituye sólo el 0,05\% (105 documentos) de i total de referencias bibliográficas que registra ISA en el periodo y sólo el 0,2\% (342 documentos) de lo que registra LISA. El primer documento registrado por ISA aparece en 1967, mientras que el primero que registra LISA sobre los temas de nuestro interés procede de 1978. Es en la década de 1980-89 cuando el tema parece tomar fuerza y aparece un número más significativo de publicaciones.

\section{En la literatura sobre informática}

Al analizar la presencia de los temas sobre inteligencia organizacional en la literatura científica de informática, a partir de lo que recoge Computer SeleCt3@ se encuentra que en el trimestre marzo-mayo de 1998, esa literatura cubre más de 600 documentos sobre el tema bajo estudio, lo cual constituye alrededor de $0,3 \%$ de total de documentos incluidos en la base de datos. Es decir, el tema de la inteligencia organizacional tiene más presencia en la literatura sobre informática correspondiente a 3 meses de publicacion, que en la literatura sobre ciencias de la información en cerca de 30 años. Esto lleva a pensar que la presencia de] tema en el desarrollo informática es relativamente alta, lo cual puede comprobarse si complementariamente se estudia el universo de productos informáticos para inteligencia organizacional o relacionados con ella.
Tomemos el caso de los productos de software. En particular, si se estudia el comportamiento de la presencia de productos como los paquetes de programas para procesamiento analítico en linea (on line analytical processíng, OLAP), los sistemas de apoyo a la toma de decisiones (decision support systems, DSS), los sistemas de información ejecutiva (executive ínformation systems, EIS) y los llamados almacenes de datos (data warehouses), que son las herramientas informáticas más difundidas para el tratamiento de la información con vistas a la inteligencia organizacional, se encuentra lo siguiente: en el semestre diciembre97 a mayo-98, Computer Select presenta un total de más de 43000 productos de software. De ellos, el $0,27 \%$ (116 productos) en la categoría de OLAP, el $0,32 \%$ (135 productos) son DSS, $0,18 \%$ (58 productos) en la categoría de EIS y el 0,39\% (170 soluciones) en la de almacenes de datos.

Aun teniendo en cuenta que generalmente los sistemas mencionados no se usan aisladamente sino que se complementan unos a otros, es obvio que existen muchas más soluciones informáticas (502 hasta mayo de 1998) y más publicaciones sobre estas soluciones (600 en la misma fecha), que las publicaciones sobre los aspectos conceptuales y métodológicos de la inteligencia organizacional, as i como sobre experiencias, estudios de caso y otros tipos de documentos que arrojen luz sobre el tema en cuestión, más que sobre las soluciones informáticas para el mismo.

Todo lo anterior explica la aseveración de Thomas D. Walker: "La literatura relativa a inteligencia competitiva radica principalmente fuera de las ciencias de la información y la bibliotecologia. Ya que los temas relevantes no están claramente definidos y porque la términologia es usada confusamente, el acceso bibliográfico se hace difícil". 


\section{En la prensa internacional}

Un enfoque complementario para conocer la presencia de la inteligencia organizacional en las fuentes de información, que permita hacerse una idea de su presencia real en el mundo gerencia;, se obtiene analizando la información de prensa. Si se observa el comportamiento de la información de prensa internacional (excluyendo Estados Unidos) respecto a los temas de la inteligencia corporativa, la inteligencia organizacional y los temas relacionados, a partir de lo indizado por F\&S Index Plus TeXt4s, en el periodo 1994-97, se encuentra que el uso fundamental se dirige a la obtención de información de mercado y que la mayor parte de las noticias se refieren al uso de las tecnologías de información. (Resulta interesante que en un caso, el tema es el espionaje industrial, lo que confirma lo dicho antes con respecto a la confusión reinante respecto al contenido preciso de la inteligencia organizacional.)

En cuanto a los paises donde se originan las noticias, se destaca Gran Bretaña, seguida por Canadá. Es interesante que en tres de los llamados "tigres asiáticos" (Singapur, Malasia y Corea del Sur) también se originan noticias sobre el tema y que Estados Unidos, aunque no fue objeto de la búsqueda de información, aparece en un porcentaje significativo de noticias, debido a la presencia de sus empresas en los negocios internacionales.

Lo anterior parece confirmar la aseveración de Ben Barnes, Gerente General de Business Intelligence Solutions, de IBM, en cuanto a la globalización de las soluciones informáticas para inteligencia organizacional, lo que haría de esta esfera de actividad un negocio de más de 70 mil millones de dólares al principio del próximo siglo, convirtiéndose la inteligencia organizacional en una actividad global más importante que la de j resto de los sistemas informáticos tradicionales. (Stodder, 1998)
Hasta aquí, todo parece indicar que la inteligencia organizacional se está convirtiendo más en un tema de la informática que de las ciencias de la informaci $i)$ n. Aparentemente, lo que está ocurriendo es que por su esencia e instrumentos, la informática está en condiciones de dar soluciones rapidas a problemas más o menos parciales, mientras que la conceptualización de una actividad nueva dentro de las ciencias de la información es una tarea en si misma compleja. Esta complejidad crece en la medida en que por una parte esta nueva actividad se mezcla, se orienta y depende fundamentalmente de las ciencias gerenciales y por otra parte, surgen continuamente enfoques teóricos muy diferentes. Dos e emplos de ello son los siguientes:

- el enfoque de las organizaciones de inteligencia de fuentes diversas (open source íntelligence organizations, OSI) como mecanismo organizativo y métodológico y

- el de la gestión de j conocimiento mediante herramientas informáticas.

Un examen superficial de la literatura sobre informática ofrece cientos de productos de software y de artículos científicos sobre el uso de herramientas informáticas para la gestión de conocimientos. Si se observan las definiciones que algunos autores ofrecen sobre la gestión de conocimientos, se evidencia una confusión similar a la que existe con los términos relacionados a la inteligencia organizacional, e incluso discusiones estériles sobre el verdadero significado de la expresión. Dos de los más claros enfoques son los siguientes:

"La informática nos ha dado acceso a todo tipo de información, pero demasiada información puede algunas veces ser tan limitante como información insuficiente si usted no puede usarla para resolver un problema u obtener un beneficio. La necesidad de convertir información en conocimiento ha generado una multitud de herramientas de gestión de conocimientos y de empleados cuyo trabajo es tomar una masa abrumadora de datos y hacerlos tangibles, accesibles y útiles". (Miliman, H. 1997)

En principio, esta definición implícita y algo ambigua de la gestión de conocimientos coincide en lo esencial con la definición de la inteligencia organizacional. La diferencia principal radica en que mientras para los sistemas de inteligencia organizacional se proponen fundamentalmente herramientas informáticas que contribuyen a dar solución a los problemas mediante el análisis de la información, proceso en que el factor humano es determinante, los productores de software para gestión de conocimientos dicen tener la solución completa de i problema. Obsérvese, no obstante, la similitud de esta definición con la de j concepto de valor añadido de Taylor, en el sentido de que el valor añadido en bibliotecología es un conjunto de procesos para convertir datos útiles en conocimientos productivos. (Taylor, R. S. 1982).

Sin embargo, una definición más precisa nos da Jeff Angus:

"El problema de negocios que la gestión de conocimientos debe resolver es que el conocimiento adquirido mediante la experiencia no se vuelve a usar porque no se comparte de manera formal. Ya sea para evitar repetir errores, para asegurar la utilización de las mejores prácticas ya probadas o simplemente para conocer lo que los empleados han aprendido sobre suministradores, clientes o competidores, la gestión de conocimientos es el concepto bajo el cual la información se convierte en conocimiento activo y se hace disponible fácilmente, de modo utilizable por las personas que pueden aplicarlo. La gestión de conocimientos es una manera de hacer negocios. En realidad, es más una práctica de negocios que un producto. Los productos (de informática) son los que facilitan la práctica de la gestión de los conocimientos - 0 al menos facetas específicas de él - con el uso apropiado de las tecnologías de la información" (Angus, J., 1998). 
El enfoque de Angus permite una diferenciación más clara entre la gestión de conocimientos y la inteligencia organizacional, evidenciando que la primera puede ser utilizada por la segunda y que las herramientas informáticas de la gestión de los conocimientos pueden ser también usadas para los propósitos de la inteligencia organizacional.

Con respecto a las $\mathrm{OS}_{i}$, Fedanzo (Fedanzo, A., 1997) las plantea como un modelo mejorado de los métodos actuales de inteligencia, que no cambia la esencia de la actividad sino sus métodos. El modelo consiste en "una confederación de participantes que trabajan bajo consenso con control central mínimo y máxima cooperación". Es decir, no hay un "cerebro" único que recopile, organice, analice y distribuya la información. Los participantes no trabajan en un lugar único y lo que se reúne son sus contribuciones listas para el uso. Se refiere a la imagen de un erizo, en lugar de a la tradicional organización piramidal. Cada proyección del erizo es más bien una proyección del sistema, un posible nodo donde se reciben solicitudes y se ofrecen soluciones. Por ello, más que un coordinador, es necesario un cuerpo de coordinación. Los solicitantes pueden ser también participantes de esta organización y ofrecer sus contribuciones, segun sea el caso. En esencia, una OS $S_{i}$ seria más bien un mecanismo de coordinación de las actividades de inteligencia que otros realicen. El principio guia de este enfoque es que "las personas saben más que lo que la informática será capaz de emular mediante sus algoritmos de búsqueda en el futuro previsible". (Fedanzo, A., 1997). Según Fedanzo, no es presumible que la tecnología satisfaga los requerimientos de las $\mathrm{OS}_{i}$ si los sistemas de gestión de información de éstas se basan en grandes bases de datos. Ello es así, segun ese autor, porque si los factores limitantes no fuesen los problemas de almacenamiento y acceso a los datos, serían entonces los de entrada y actualización de los mismos. Por tanto, el enfoque $\mathrm{OS}_{i}$ pretende convertir el obstáculo que constituyen la heterogeneidad de fuentes y recursos de información en una fortaleza, a partir de un cambio de método y de mayor participación humana.

Respecto a lo anterior, los productores de herramientas informáticas para la inteligencia organizacional pudíesen argumentar que ya existen suficientes soluciones de ese tipo como para demostrar que la tecnología sí puede satisfacer las necesidades de las OS $\mathrm{S}_{\text {. }}$ Pero precisamente la profusion de nuevas soluciones y la necesidad imperiosa de utilizar personal calificado que complemente los resultados de esas herramientas con análisis basados en criterios de experto, indica que las OS $i$ son una opción inteligente, que no niega el uso de diversas herramientas informáticas y de hecho el necesario complemento entre ellas.

\section{LA DISYUNTIVA DE LA INTELIGENCIA ORGANIZACIONAL: CENTRALIZAR O DESCENTRALIZAR?.}

Aqui se presenta una disyuntiva para las entidades que desean implantar un sistema de inteligencia organizacional. Debe ser el sistema centralizado, como el que funciona cuando se posee un gran almacen de datos con información de todas las áreas de la entidad, o por el contrario es mejor un enfoque tipo OSI?

En realidad, la disyuntiva es aparente. Una de las principales características de los sistemas de gestión de información es que deben adaptarse a la estructura y esquemas organizativos de la institución a la cual sirven. Los sistemas de inteligencia, en tanto que sistemas de gestión de información, no escapan a esa exigencia sino que por el contrario, en esos casos ella se hace más fuerte, debido a las expectativas que se tienen sobre los mismos.

Existen casos particulares en que la solución única en un sentido u otro es evidente. Por ejemplo, en el caso de empresas con líneas homogéneas de producción dentro de un sector, los almacenes de datos y los OLAP como complemento, pueden ser una solución ideal. Sin embargo, en grupos empresariales con producciones diversas pero donde la toma de decisiones gerenciales y financieras es centralizada, la solución mencionada puede ser de dificil implantación y se requiere de otras herramientas y de mayor concurso humano. Por otra parte, en los casos en que elementos informativos muy dispares, como por ejemplo la investigación-desarrollo, la formación de recursos humanos y la actividad legal (es decir, donde se maneja información preponderantemente textual y cualitativa, ademas de información financiera y de mercado), sean algunos de los componentes importantes de las decisiones finales, es presumible que mecanismos como el de las OSi sean más apropiados. Un ejemplo de ello es el de la industria biofarmacéutica.

\section{EL PAPEL DE LA INFORMACIÓN DE INTELIGENCIA EN LA INDUSTRIA BIOFARMACÉUTICA}

"'Biotecnología... la definición que a largo plazo puede resultar más descriptiva, en relación con la economía mundial, es... hacer dinero con la biología". Coíwell y Sasson, World Science Report, 1997-98.

La industria biotecnológica, dedicada al desarrollo de nuevas tecnologías y procesos, es resultado de la aplicación de los conocimientos y descubrimientos de la biología, la bioquímica y la fisiología entre otras ciencias. A la vez, los conocimientos biotecnológicos son aplicables a diversas industrias, como la farmacéutica, la agrícola, la medioambiental, la industria metalúrgica, la petrolera y otras.

La industria biofarmacéutica es el segmento de la industria farmacéutica que sustenta el desarrollo de medicamentos mediante la aplicación de técnicas biotecnológicas. Por tanto, forma parte también de la industria biotecnológica. (Consultoría Biomundi, 1997). 
De modo que por su origen científico y tecnológico, la industria biofarmacéutica es completamente multidisciplinaria, as i como lo es por sus aplicaciones. Por otra parte, desde el punto de vista de sus mecanismos gerenciales y sus estrategias de desarrollo, fundamentalmente a partir de los años 90, la integración es un signo distintivo de esta industria, ya sea mediante alianzas, colaboración, licenciamiento, mecanismos de armonización, producción compartida, investigación por contrato y muchas otras modalidades de desempeño, que obligan tanto al conocimiento cabal de las contrapartes comerciales, los aliados, y los clientes, como al profundo conocimiento científico para la obtención de nuevos medicamentos, as i como para el desarrollo de sus bases tecnológicas, el conocimiento de las nuevas tecnologías ajenas, los mecanismos regulatorios y otros muchos elementos de información, que hacen que esta industria sea particularmente intensiva en información, tanto en los procesos de creación de valor, como en los productos y servicios que resultan de; complejo proceso de investigaciónproducción-comercializaci ¿)n.

Desde el punto de vista de j uso de la información por esta industria, su necesidad de mecanismos de inteligencia empresarial se evidencia cuando se conoce que la industria biofarmacéutica:

- es multidisciplinaria;

- es multifactorial;

- es multinacional;

- tiene fuerte raiz científica y tecnológica se orienta al mercado es de interés global;

- tiene a la vez apoyo estatal y fuerte participación privada;

- en su desarrollo participa todo tipo de instituciones: universidades, empresas, institutos;

- organizaciones regulatorias, sociales y otras muchas;

- es económicamente poderosa;
- los procesos de obtención de nuevos productos son muy costosos y muy largos;

- tiene como signo común la integración con otras industrias y entre sí.

El vínculo entre todos estos elementos y su significado quedan resumidos en las palabras de Charles Cooney:

"Ser competitivos [en la industria medico-farmacéutical es tener productos que resuelvan un problema de salud de modo seguro y eficaz ... diseño y descubrimiento de medicamentos ... ese es el futuro de la industria. Es crucial para el éxíto la velocidad con que se llegue al mercado... el desarrollo de procesos productivos efectivos ... La innovación en productos requiere innovación tecnológica. Los nuevos productos requieren nuevas tecnologías ... la conversión de ciencia en tecnología será menos una forma de arte experimental y más una actividad precisa que otorga a las firmas que la practiquen ventajas competitivas significativas"

De la cita anterior resulta evidente que la más fuerte palanca de las compañías farmacéuticas es la investigación, y que es ahí donde se pone el mayor esfuerzo de inteligencia, ya que se depende de nuevos productos para mantener la competitividad, lo cual también enfatizan Esposito \& Gilmont (Esposito \& Gilmont, 1991).

Este criterio se reafirma por Krol, Coleman y Bryant (Krol, Coleman y Bryant, 1996) cuando llegan incluso a proponer un concepto nuevo: inteligencia competitiva científica, con el que designan el proceso de monitoreo de la investigación y de la linea de productos de los competidores, as i como las tendencias científicas y tecnológicas del sector. Aunque los autores mencionados diferencian la inteligencia competitiva científica de la inteligencia empresarial que ellos llaman tradicional, por cuanto incluyen como beneficiarios a un amplio grupo de figuras en la empresa, como la alta dirección, los grupos de desarrollo estratégico, los grupos comercial y de marketing y la actividad de investigación-desarrollo, en realidad su concepto abarca varios de los campos que el autor de este trabajo considera como dentro de la inteligencia empresarial. Acertadamente, Krol, Coleman y Bryant entienden que el impacto de la inteligencia competitiva científica es multifactorial e involucro a muchos grupos dentro de la organización. Sin embargo, esta no es la situación general de la industria farmacéutica. Una encuesta realizada por Esposito \& Gilmont a un grupo de grandes empresas europeas, japonesas y estadounidenses, que incluia varias del sector farmacéutico, dió como resultado que las empresas farmacéuticas encuestadas asignaban gran importancia a la inteligencia competitiva. El $80 \%$ de ellas tenía programas estructurados de inteligencia competitiva, aunque con bajo nivel de esfuerzo presupuestario y sólo el $40 \%$ había definido la dirección y coordinación de sus esfuerzos.

En la misma investigación, se observó cómo diferian los niveles de compromiso y los estados de implantación entre diferentes compañías, desde departamentos formales que reportaban a un vicepresidente, hasta esfuerzos ad-hoc sin formalización, pasando por mecanismos descentralizados que incluían el análisis de información por parte de los científicos. Un ejemplo más reciente, es el de la compañía Giaxo-Wellcome (Reino Unido), que ha implantado un sistema de almacen de datos, fundamentalmente para el seguimiento de funcionamiento de su cadena de creación de valor, desde la compra de materias primas hasta el embarque de los productos terminados. (Marshali, M. 1997). En este caso, el uso de los almacenes de datos es complementario al de otras herramientas de la informática para el intercambio y análisis de informaci $i$ )n científica y tecnológica, como la red que maneja el Departamento de Servicios de Información de Glaxo Group Research Ltd., el cual ha jugado un papel esencial en el éxito de la compañía. (Coutin, A. 1995). 
Independientemente de lo que hacen internamente las grandes compañías de $i$ sector farmacéutico y de $i$ biofarmacéutico en cuanto a inteligencia empresarial, lo cierto es que la industria de la información produce una gran cantidad de recursos que sirven de herramientas para esos propósitos, en particular en cuanto a fuentes de información, ya que existen cientos de bases de datos que cubren las disciplinas nutrientes de la biotecnología y las relacionadas con la Industria médico-farmacéutica, as j como decenas de miles de títulos de revistas y muchos otros tipos de documentos.

Sin embargo, no se puede decir lo mismo respecto a herramientas de software de aplicación directa en esfuerzos de inteligencia empresarial para esas industrias, sino que se utilizan herramientas de carácter general con las adaptaciones convenientes al caso. Tampoco aparece mención en la prensa internacional a aplicaciones como las que nos ocupan. En el caso de la literatura sobre ciencias de la información, es despreciable la cantidad de documentos referenciados (8 de casi 200000) que tratan sobre los temas de inteligencia y hacen alguna referencia a la biotecnología y la industria médico-farmacéutica, segun resulta de búsquedas en ISA Plus.

\section{LA INTELIGENCIA EMPRESARIAL EN LA INDUSTRIA BIOFARMACÉUTICA CUBANA: CONSULTORÍA BIOMUNDI}

La Consultoría Biomundi es una

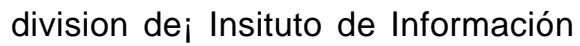
Científica y Tecnológica, del Ministerio de Ciencia, Tecnología y Medio Ambiente de la República de Cuba. Se fundó en 1992 como organización encargada de ofrecer servicios de información para la toma de decisiones en el sector biotecnológico y medico-farmacéutico.
El sector biotecnológico y médicofarmacéutico cubano está compuesto por unas 200 organizaciones dispersas por todo el país, que atienden los diferentes aspectos de la biotecnología, como biotecnología de salud humana, biotecnología agrícola (vegetal y de salud animal), biotecnología industrial y aplicaciones de la biotecnología a los estudios de medio ambiente. Ese grupo de instituciones cubre universidades, institutos de investigación, grupos de investigación en hospitales y clínicas, empresas productoras, empresas comercializadoras, órganos regulatorios, y otros. Este conjunto de organizaciones elabora más de 1500 productos, edita unas 50 publicaciones y crea alrededor de 30 bases de datos. En conjunto, prestan unos 900 servicios diferentes. En el país existe representación de más de 40 empresas extranjeras de i sector, fundamentalmente europeas y latinoamericanas. (Marín, L. 1997)

El más notable foco cubano en el sector de la biotecnología es el Polo Científico de Oeste de La Habana, que agrupa a unas 40 organizaciones entre las cuales se encuentran los más importantes líderes de i país en investigación-desarrollo, producción y comercialización, as i como hospitales, facultades e institutos universitarios y los órganos regulatorios nacionales.

El Polo Cientifico de Oeste de La Habana es un mecanismo de coordinación estratégica que facilita la ejecución de las líneas y programas de investigación de j sector, en su vinculación con los objetivos de desarrollo nacional y su ciclo productivo y de comercialización, en temas como biotecnología e ingeniería genética, química farmacéutica, investigación y producción de vacunas humanas y animales, neurociencias, técnicas de diagnóstico, medicina tropical y otros temas de punta en la investigación internacional.

En ese contexto científico-productivocomercial, surge Consultoría Biomundi, que ofrece un conjunto de servicios y productos de inteligencia organizacional. Los servicios y productos de inteligencia de Consultoría Biomundi se orientan tanto a la inteligencia en investigación-desarrollo, como a los aspectos productivos y comerciales de la biotecnología y la industria médico-farmacéutica y otros sectores relacionados. También se brinda servicio a otros sectores, como la industria de la información, la agricultura, el medio ambiente y otros.

\section{Servicios y productos de inteligencia de Consultoría Biomundi}

Consultoría Biomundi ofrece un grupo de servicios y productos de inteligencia, entre los que se pueden citar los siguientes:

- identificación y comprobación de proveedores, competidores, clientes, y otros contactos de interés,

- elaboración de perfiles de compañía, de personalidad, de productos, de mercado y de paises,

- estudios de mercado,

- estudios de tendencias de desarrollo,

• evaluación de negociaciones,

- elaboración de los perfiles corporativos de todas las organizaciones del sector en Cuba y edición del directorio correspondiente (Biociencias en Cuba),

- elaboración de los perfiles corporativos de miles de organizaciones del sector en más de 130 paises y edición del directorio correspondiente (Biomundi Internacional Directory),

- compilación de noticias sobre el desarrollo del sector en Cuba y creación de la base de datos correspondiente,

- estudios sobre el impacto de la ciencia cubana en el exterior,

- determinación de posibilidades de publicaci i)n, participación en congresos, etc.,

- auditoría de información,

- coordinación de la intranet corporativa del Polo Científico del Oeste. 
Además se ofrecen otros servicios propios de la gestión de información, como:

- búsquedas de información a solicitud,

- creación de bases de datos,

- monitoreo de información,

- boletín de alerta informativa sobre la biotecnología en el mundo,

- consulta a bases de datos,

- cursos y entrenamientos sobre gestión de información, inteligencia organizacional, creación de bases de datos, gestión de tecnología de la información, automatización de bibliotecas, Internet, análisis de información, el valor agregado en la gestión de información.

\section{Recursos para la inteligencia organizacional en Consultoría Biomundi}

Como parte de la gesti i)n de información, los recursos fundamentales de la inteligencia organizacional, son los siguientes: e la concepción y el método organizativo, o el factor humano,

- las fuentes de información,

- la tecnología y

- el sistema de relaciones y comunicación.

Más detalladamente lo veremos a continuación:

\section{Concepción de trabajo y método organizativo}

Consultoría Biomundi es una entidad de inteligencia organizacional com actividad centralizada, formal y permanente, que trabaja para un sector en lugar de trabajar para una única organizacion, a diferencia de los sistemas tipicos de inteligencia empresarial, que trabajan internamente para la organización que financia sus actividades. Por ello, su politica comercial es a la vez la de una consultaría y de un centro de información. Se ofrecen algunos servicios permanentes y otros a solicitud de usuarios, con relaciones de cobro y pago según el caso, y servicios gratuitos en algunas ocasiones. Se utiliza el "soft-data" según las necesidades de i producto o servicio solicitado, pero no se formaliza en bases de datos. Se utiliza tanto información externa como interna de i sector de atención. La concepción permite que tanto organizaciones de pequeña empresa como no lucrativas, tengan acceso a servicios de inteligencia organizacional, a diferencia de la concepción en la cual estos servicios se organizan por las organizaciones que son capaces de sostenerlos. Por su concepcion, Consultoría Biomundi evoluciona hacia el estatus de organización abierta de inteligencia organizacional o, para llamarlo como Fedanzo, 'open source íntellígence organízatíon" (OS $\mathrm{O}$ ), en la que en lugar de un "cerebro" centralizado, la organizaci $i) n$ es un aparato de coordinación, con ramificaciones externas tanto en captaci $i$ )n como en análisis de información, con un mecanismo permanente de retroaiimentaci $i) n$. En el caso de Consultoría Biomundi, esto se materializa a partir de j uso de una red de consultores asociados y gracias al mecanismo de retroalimentación de las organizaciones usuarias de los servicios y productos. El factor clave de éxito de este enfoque es su confianza en el factor humano y en su capacidad de análisis, utilizando la tecnología a su servicio

\section{El factor hurnano}

El staff de Consultoría Biomundi está compuesto por unos 40 profesionales, cuya edad promedio es alrededor de los 35 años, con formación básica en ciencias farmacéuticas, biología, química, medicina, leyes, economía, ingenierías, ciencias de la información, informática y telecomunicaciones. En una concepción matricial de la organización, el staff trabaja fundamentalmente en equipo. Existen 15 gerentes de productos y/o servicios, con un enfoque horizontal de su actividad y 5 coordinadores que agrupan las gerencias, desde el punto de vista de asignación de recursos y coordinaciones externas para facilitar la planeación. Los trabajos se elaboran por equipos de 263 personas, a partir dej reconocimiento de la mayor experiencia de algunos especialistas en determinado campo pero con manejo común de las técnicas de búsqueda, procesamiento y análisis de información, obtenido fundamentalmente en estudios de post-grado y mediante la práctica diaria. Adicionalmente, se cuenta con el apoyo ocasional de los miembros de una red de consultores asociados, los cuales son utilizados mayormente sobre una base ad-honorem, en los casos necesarios.

\section{Tecnología}

Consultoría Biomundi cuenta con una red local de 42 terminales, conectada a la red pública de transmisión de datos, con salida internacional. Sobre esta red funciona una red de bases de datos en disco compacto. Según las funciones necesarias, se poseen servidores NT, UNIX, SCO y Netware de Noveli. Se trabaja en ambiente Windows y se ha normalizado el software para todas las funciones. La intranet ha permitido consolidar la cultura de intercambio de información. Actualmente se trabaja en la coordinación de la intranet corporativa de Polo Científico de; Oeste (unas 40 organizaciones), lo que facilitará la captación de información y su distribución.

\section{Fuentes de información}

Consultoría Biomundi cuenta con más de 40 bases de datos en cd-rom en las temáticas de principal interés, como medicina, biología, química, agricultura, biotecnología y ciencias farmacéuticas, directorios nacionales e intenacionales, prensa nacional e internacional, informaci $i$ )n regulatoria, información gerenciai y economía. Igualmente se cuenta con acceso a varios hosts internacionales, a Internet y con contacto con consultores nacionales y extranjeros. 


\section{Sistema de relaciones y comunicación}

Consultoría Biomundi mantiene contacto con todas las organizaciones cubanas de i sector que atiende y con cientos de organizaciones de todo el mundo. Se recibe regularmente información no publicada de miles de organizaciones (institutos, empresas, universidades y muchos otros) de más de 100 paises. Se ha desarrollado un mecanismo de intercambio bibliotecario que utiliza principamente los documentos no confidenciales que elabora la consultaría. Se tienen relaciones de colaboración con otras consultarías cubanas, asi como de América Latina y Europa.

\section{Organizational intelligence in the biopharmaceutical industry}

\begin{abstract}
An analysis of the state-of-art of organizational (entrepreneurial, corporate, competitive, economic) intelligence is presented from the point of view of its impact on the scientific literature about information science, economic press and scientific and corporate information referring to information technologies. Different focuses, apparently diverging, are discussed. Previous results related to the biopharmaceutical industry were worldwide evaluated. A discription of the methods of work and the results of the Biomundi Consultancy, as an organizational intelligence center for the biopharmaceutical industry, are presented.
\end{abstract}

\section{Keywords}

Organizational intelligence,

Biopharmaceutical industry; Scientific

literature; Information technologies.

\section{REFERENCIAS}

1. ANGUS, JEFF. Knowledge Management: Great Concept... But What Is It? Information Week. March 16, 1998 n673 p58(7).

2. COLWELL, RITA and ALBERT SASSON. Biotechnology and development, Worid Science Report, UNESCO, Paris, 199798. pp. 253-268.

3. CONSULTORÍA BIOMUNDIIIDICT. EI mundo en hechos y cifras.Farmac@ biotecnología, 1996-97. ISSN: 10272844; ISBN: 959-234-007-2

4. COONEY, CHARLES. The Art of Transiating Science into Technology. Pharmaceutical Technoloqv, 5(5):15, 1993. Citado por Alberto Nunez, ESCALADO EN LA INDUSTRIA BIOFARMACÉUTICA, Centro de Quimica Farmacéutica, La Habana, 1997, sin publicar.

5. COUTIN, A. A general overview of pharmaceutical industry and the impact of some new toois on its research activities. A practicai example using similarity search. The University of Sheffieid, Sept. 1995. No publicado.

6. DKAKI, T. and B. DOUSSET. Tetralogie: A method for competitive intelligence. Dkaki@atias.irit.fr. IRIT, Universite Paul Sabatie, Touiouse, 17 Aug., 1998.

7. DRUCKER, PETER. La información que necesitan los ejecutivos de hoy. En Managing in a time of great change. Truman Talley Books/Dutton., New York, 1995.

8. ESPOSITO, MICHAEL A. \& ERNEST R. GILMONT. Competitive Intelligence: Doing Corporate Homework, Pharmaceutical Executive, Dec. 1991, pp. 68-74.

9. FEDANZO, ANTHONY. Implementing Open Source Intelligence Through a Distributed Contribution Model. Obtenido por correo electronico, sept. 1997.

10. KROL,-T.F.; COLEMAN,-J.C.; BRYANT,P.J. Scientific competitive intelligence in R\&D decision making. Dru-qinformation-Journal-(US) Vol. 30, Issue 1, p. 243-256, Jan-Mar 1996, .MARIN, LUIS. Breve perfil de la actividad de la industria biotecnológica y farmacéutica, servicios de salud y equipos medicos en Cuba. Informe 0300, Consultoría Biomundi, La Habana, Noviembre de 1997.

11. MARSHALL, MARTIN. Giaxo fine-tunes data warehouse. Communications Week, July 14, 1997 n672 p35(2).

12. MILLMAN, HOWARD. Knowledge equais power. Infoworid. Nov 17,1997 vl 9 n46 pl 16(9).

13. OROZCO, EDUARDO. La inteligencia corporativa fuera de la gran empresa. Aceptado para publicación en Ciencias de la Información, Setiembre 1998.
14. STODDER, DAVID. Business intelligence goes global. Database Programminq \& Desiqn. April 1998, vl 1 n4 pS24(6).

15. TAYLOR, R. S. Value-added processes in the information ]ife cycle. Journal of the American Societv for Information Science, 33 (5) Sept.82, 341-346.

16. WALKER, THOMAS D. The literatura of competitive intelligence, Librarv Trends. 43(2) Fall 94, p. 271-84.

Notas

1) Infomation Science Abstracts Plus, March 1998. IFI Plenum Data Corp.

2) Library and Information Science Abstracts, June 1998. Reed Business Information Limited.

3) Computer Select, Dec. 1997-May 1998. Information Access Company.

4) F\& S Index Plus Text, Information Access Company, 1994-97

\section{CONTENIDO}

INTRODUCCióN

LA INTELIGENCIA ORGANIZACIONAL EN LOS RECURSOS MODERNOS DE INFORMAClóN

En la literatura sobre ciencias de la información.

En la literatura sobre informática.

En la prensa internacional.

- LA DISYUNTIVA DE LA INTELIGENCIA ORGANIZACIONAL: CENTRALIZAR

0 DESCENTRALIZAR?.

- El PAPEL DE LA INFORMACión DE INTELIGENCIA EN LA INDUSTRIA

BIOFARMACÉUTICA.

- LA INTELIGENCIA EMPRESARIAL EN LA INDUSTRIA BIOFARMACÉUTICA CUBANA: CONSULTORÍA BIOMUNDI.

Servicios y productos de inteligencia de Consultoría Biomundi

Recursos para la inteligencia organizacional en Consultoría Biomundi.

Concepción de trabajo y método organizativo Factor humano

Fuentes de información

Tecnología

Sistema de relaciones y comunicación.

\section{Eduardo Orozco Silva}

Consultoría Biomundi/IDICT

Calle 200 No. 1922, Reparto Atabey, Playa, Ciudad de La Habana, Cuba

Teléfonos: 53-7-212823, 217711, 217708

Fax: 53-7-336529, 338237

Correo electrónico: orozco@biomundi.inf _.. 